High-throughput and high-efficient micro-solid phase extraction based on sulfonatedpolyaniline/polyacrylonitrile nanofibers mat for determination of fluoroquinolones in animal-original foods Ningge Jian ${ }^{\mathrm{a} \dagger}$, Meng Zhao ${ }^{\mathrm{a} \dagger}$, Sihui Liang ${ }^{\mathrm{a}}$, Jiankun Cao ${ }^{\mathrm{a}}$, Chunmin Wang ${ }^{\mathrm{b}}$, Qian $\mathrm{Xu}^{\mathrm{a}^{*}}, \mathrm{Jian} \mathrm{Li}^{\mathrm{b} *}$

${ }^{a}$ Key Laboratory of Environmental Medicine Engineering, Ministry of Education, School of Public Health, Southeast University, Nanjing 210009, China

b Suzhou Municipal Center for Disease Prevention and Control, Suzhou 215004, China

\title{
*Corresponding to:
}

Qian Xu. Tel.: +86025 83272563; E-mail: q_xu68@163.com, q-xu@seu.edu.cn; fax: +86 025 83204231.

Jian Li. Tel.: +86 18962168852; E-mail: 1055729273@qq.com.

\section{Text S1 Chemicals and reagents}

Aniline, $m$-sulfonate aniline were purchased from Greagent (ShangHai, China). Polyacrylonitrile (PAN, average MW 150,000) was supplied by Macklin (ShangHai, China). $\mathrm{Na}_{2} \mathrm{HPO}_{4} \cdot 12 \mathrm{H}_{2} \mathrm{O}$, citric acid monohydrate and $\mathrm{Na}_{2}$ EDTA used for preparing EDTA-McI1vaine's buffer were from Sinopharm (ShangHai, China). Target FQs, including Norfloxacin (NOR), Enrofloxacin (ENR), Lomefloxacin (LOM), Pefloxacin (PEF), Ciprofloxacin (CIP) and Ofloxacin (OFL), were purchased from Sigma-Aldrich (purity $>99.0 \%$, St. Louis, MO, USA). HPLC grade methanol was from Merck KGaA (Darmstadt Germany). All other reagents and chemicals used in the experiments were from Chemical Reagent Factory (Shanghai, China). Ultrapure water was obtained from Milli-Q (Millipore, MA, USA) water purification system. The $\mathrm{pH}$ values of both the 
solutions were adjusted using $1.0 \mathrm{~mol} \cdot \mathrm{L}^{-1} \mathrm{HCl}$ or $\mathrm{NaOH}$ solution.

The extraction solvent (EDTA-McIlvaine's buffer) was prepared by dissolving $2.1 \mathrm{~g}$ of citric acid monohydrate and $4.48 \mathrm{~g}$ of $\mathrm{Na}_{2} \mathrm{HPO}_{4} \cdot 12 \mathrm{H}_{2} \mathrm{O}\left(10 \mathrm{mmol}\right.$ citric acid/12.5 mmol $\left.\mathrm{Na}_{2} \mathrm{HPO}_{4}\right)$ in $162.5 \mathrm{~mL}$ of purified water. After the $\mathrm{pH}$ was adjusted to $4,6.05 \mathrm{~g}$ (18 mmol) of Na2EDTA was added to the solution and stirred to dissolve.

\section{Text S2 Preparation of NFsMs (raw PAN NFsM, PANI/PAN NFsM and sulfonated-PANI/PAN}

NFsM).

Briefly, $3 \mathrm{~g}$ PAN was dissolved in $20 \mathrm{~mL}$ DMF and stirred at room temperature for $5 \mathrm{~h}$ to obtain a $15 \%(\mathrm{w} / \mathrm{v})$ homogeneous solution. Then, the homogeneous mixture was loaded into a syringe with uniform diameters of $0.5 \mathrm{~mm}$ and driven by a syringe pump with a flow rate of $1 \mathrm{~mL} \cdot \mathrm{h}^{-1}$. The distance between the collector and the spinneret was $15 \mathrm{~cm}$. The NFsM was collected on aluminum foil at a high voltage of $18 \mathrm{kV}$.

For PANI/PAN NFsM, $0.02 \mathrm{~mol}$ aniline were added in $100 \mathrm{~mL} 0.2 \mathrm{~mol} \cdot \mathrm{L}^{-1} \mathrm{HCl}$ solution to get solution A. Then, $2.28 \mathrm{~g}\left(\mathrm{NH}_{4}\right)_{2} \mathrm{~S}_{2} \mathrm{O}_{8}(0.01 \mathrm{~mol})$ was dissolved in $80 \mathrm{~mL}$ water to get solution $\mathrm{B}$. After solution A and solution B was mixed together in a reaction vessel under sonication, the $\mathrm{pH}$ of the mixture was adjusted to 2. Then, the raw PAN NFsM was immersed in the solution. After 12 hours at $4{ }^{\circ} \mathrm{C}$, PANI/PAN NFsM were obtained by washing with water repeatedly until the solution turned colorless, followed by vacuum drying at $60{ }^{\circ} \mathrm{C}$ for $5 \mathrm{~h}$.

As for sulfonated-PANI/PAN NFsM, $0.01 \mathrm{~mol}$ aniline and $0.01 \mathrm{~mol} m$-sulfonate aniline were used as monomers for polymerization. The following steps were the same with that of PANI/PAN NFsM. 
(SEM, S-4800, Hitachi, Japan) and transmission electron microscopy (TEM, JEOL, Japan). The average diameter of fibers was determined using Image $J$ software (National Institutes of Health, USA). The specific surface areas and pore parameters of NFsMs were measured by nitrogen sorption porosimetry performed on an ASAP 2020 Accelerated Surface Area and Porosimetry Analyzer (Micromeritics Instrument Corporation, Norcross, GA). The specific surface area was calculated using the BET equation and the mesopores size distribution was determined by BarrettJoyner-Halenda (BJH) method. Fourier transform infrared (FT-IR) spectra was obtained by FT-IR Spectrophotometer (IS10 spectrometer, Nicolet, USA) over the wave number range of 400-4000 $\mathrm{cm}^{-1}$ to analyze functional groups in NFsM.

\section{Text S3 UPLC-MS/MS analysis}

The detailed mass spectrometer operating conditions were as follows: capillary voltage $3.0 \mathrm{kV}$, desolvation temperature $350{ }^{\circ} \mathrm{C}$ and desolvation gas $800 \mathrm{~L} \cdot \mathrm{hr}^{-1}$.

An Acquity UPLC BEH C18 $(2.1 \mathrm{~mm} \times 50 \mathrm{~mm}, 1.7 \mu \mathrm{m})$ cartridge was used for the separation of the target FQs at $35^{\circ} \mathrm{C}$. The injection volume was $5 \mu \mathrm{L}$. Solvent A (water with $0.2 \%$ formic acid) and solvent $\mathrm{B}$ (acetonitrile) were used as the mobile phase at a flow rate of $0.25 \mathrm{~mL} \mathrm{~min}^{-1}$. The gradient was set as follows: solvent B stayed at $15 \%$ in the first $2.0 \mathrm{~min}$ and increased from $15 \%$ to $90 \%$ linearly from 2.0 to $2.5 \mathrm{~min}$. From 3.5 to $4.5 \mathrm{~min}$, B decreased from $90 \%$ to $15 \%$. Then B remained at $15 \%$ from 4.5 to $5.0 \mathrm{~min}$.

\section{Text S4 The LODs and the LOQs determined by the method provided by Har ris.}

(1) After estimating the detection limit from previous experience with the method, prepare a sample whose concentration is 1 to 5 times the detection limit. 
(2) Measure the signal from $n$ replicate samples $(n \geqslant 7)$.

(3) Compute the standard deviation $(s)$ of the $n$ measurements.

(4) Measure the signal from $n$ blanks (containing no analyte) and find the mean value, $\mathrm{y}_{\text {blank }}$.

(5) The minimum detectable signal, $y_{d l}$, is defined as

(6) The corrected signal, $\mathrm{y}_{\text {sample }}-\mathrm{y}_{\text {blank }}$, is proportional to sample concentration:

Calibration line: $y_{\text {sample }}-y_{\text {blank }}=m \times$ sample concentration<smiles></smiles>
curve. The minimum detectable concentration, also called the detection limit, is obtained by substituting $y_{d l}$ from Equation (1) for $\mathrm{y}_{\text {sample }}$ in Equation(2):

Detection limit: Minimum detectable concentration $=3 \mathrm{~s} / \mathrm{m}$

Quantitation limit: Lower limit of quantitation $=10 \mathrm{~s} / \mathrm{m}$
1 polymerization (Different molar ratios $(10: 0,9: 1,8: 2,7: 3,6: 4,5: 5,4: 6 \ldots)$ of $m$-sulfonate aniline to aniline were performed for the preparation of sulfonated-PANI/PAN NFsM. The polymerization 

introduced the most sulfonic acid groups in the sulfonated-PANI/PAN NFsM).

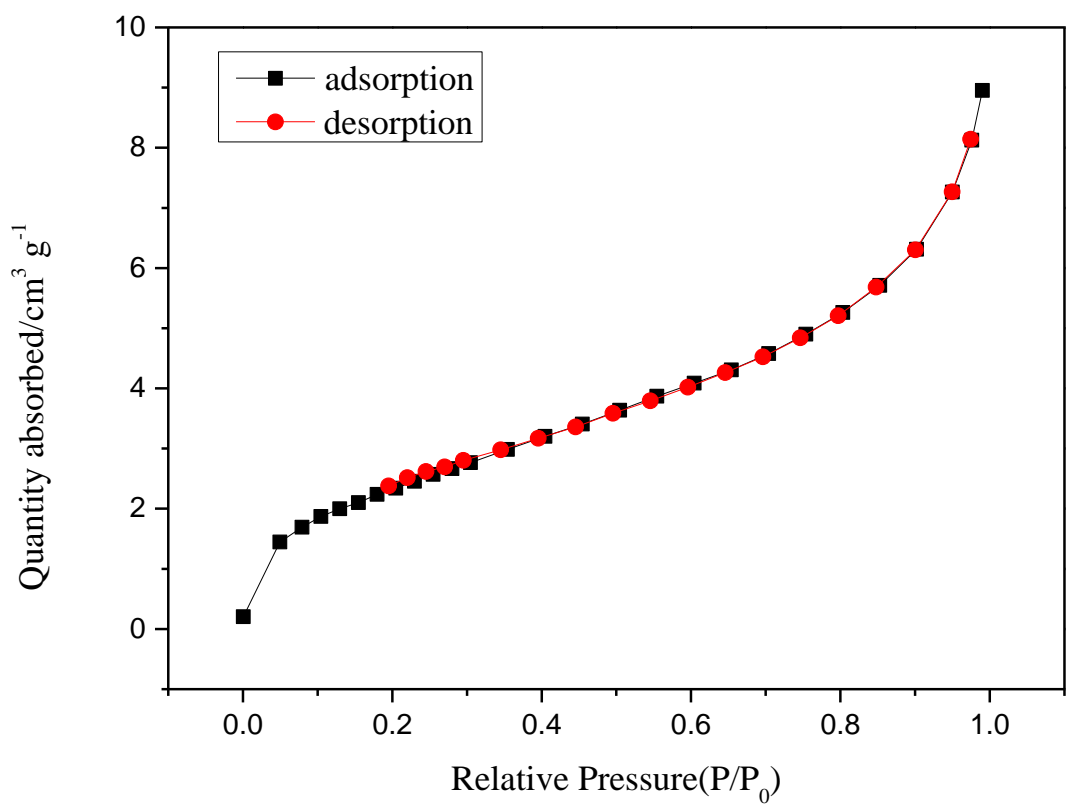

reaction occurred only when at least half of the aniline was presented. The 1:1 molar ratio of msulfonate aniline to aniline not only ensured the occurrence of the polymerization reaction, but also
89

Figure $\mathrm{S} 1 \mathrm{~N}_{2}$ adsorption-desorption isotherms for the sulfonated-PANI/PAN NFsM.

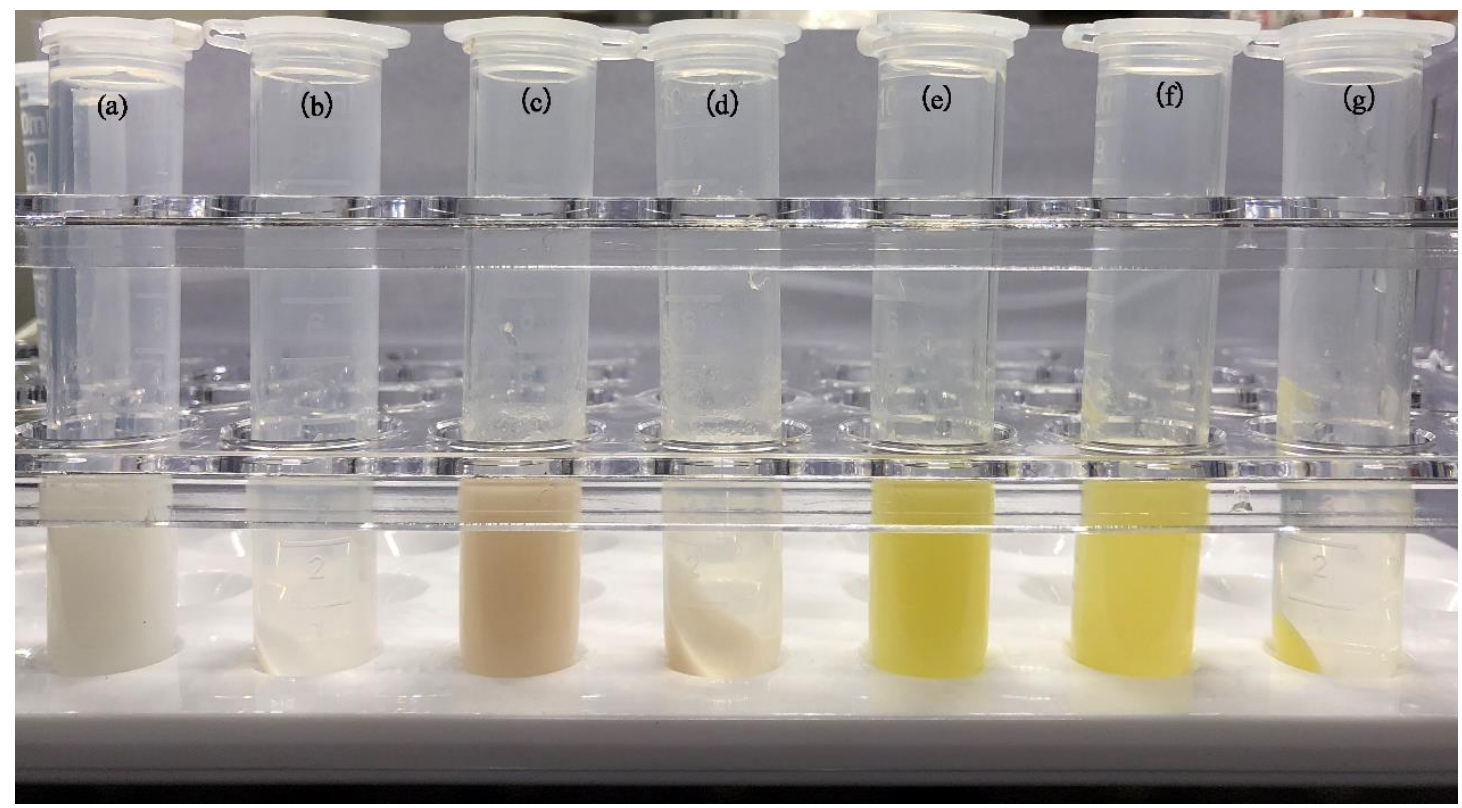

Figure S2 Figures of sample extracts before and after centrifugation. (a) milk in $2.5 \mathrm{~mL}$ EDTAMcIlvaine's buffer (b) supernatant of milk extract (c) meat in 2.5 mL EDTA-McIlvaine's buffer (d) supernatant of milk extract (e) egg in 2.5 mL EDTA-McIlvaine's buffer (f) supernatant of egg extract 
(a)
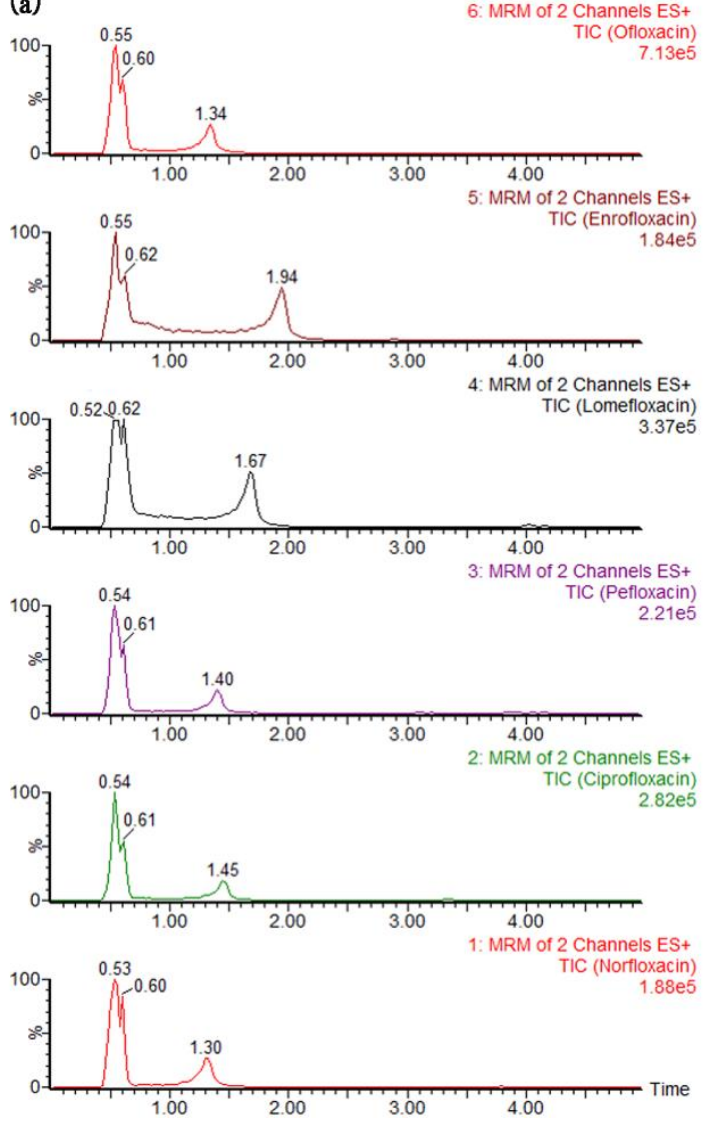

(b) 6: MRM of 2 Channels ES+
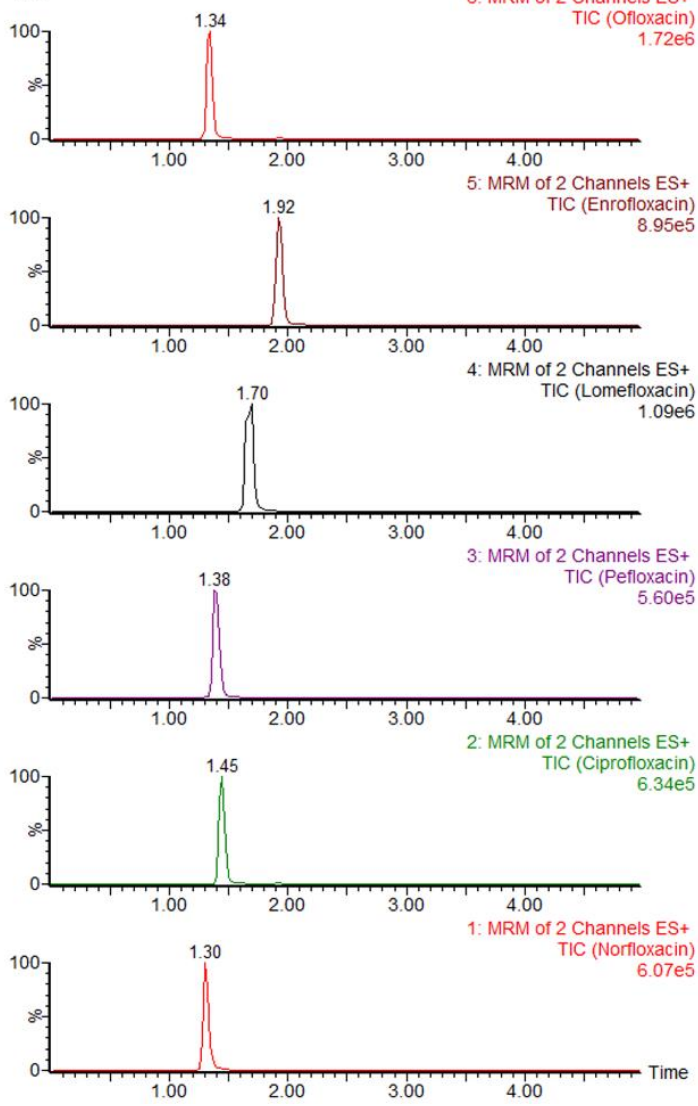

97

Figure S3 Typical extracted ion chromatograms (EIC) and MS/MS spectra of FQs obtained in (a) $10 \%$ formic acid/acetonitrile (b) $0.2 \%$ formic acid/water.

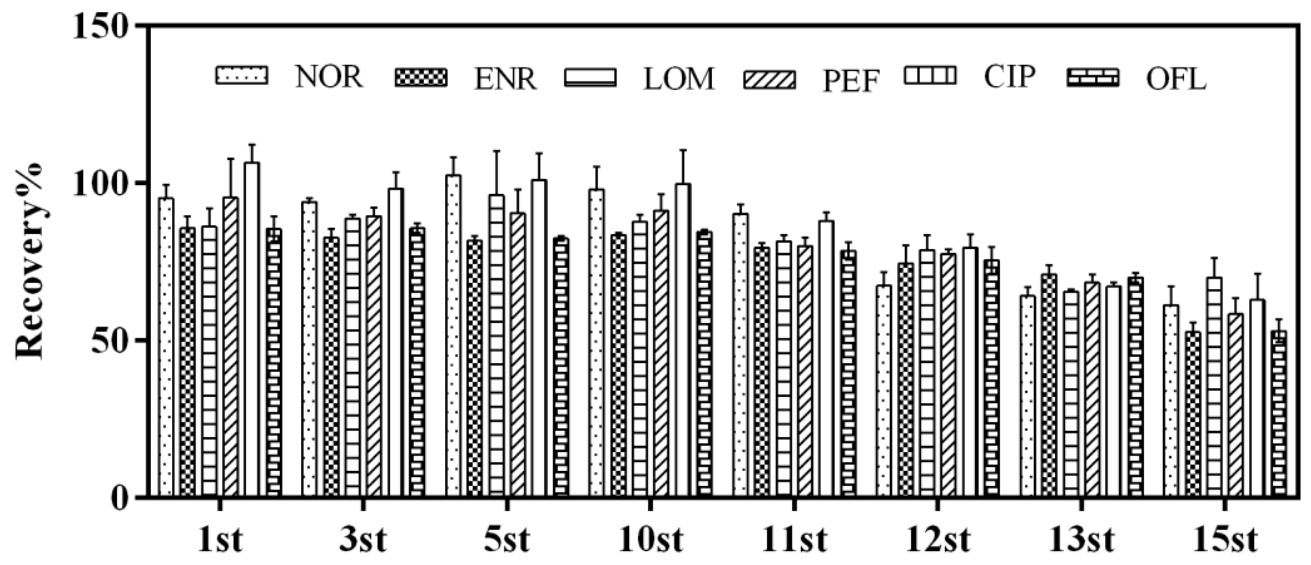

Figure S4 Reusability of sulfonated-PANI/PAN NFsM. 
Table S1 The optimized MS/MS conditions used in this work

\begin{tabular}{|c|c|c|c|c|c|c|}
\hline Name & $\mathrm{pK}_{\mathrm{a} 1}$ & $\mathrm{pK}_{\mathrm{a} 2}$ & Parent ion & Daughter ion & $\begin{array}{c}\text { Cone voltage } \\
\text { (V) }\end{array}$ & $\begin{array}{c}\text { Collision Energy } \\
(\mathrm{eV})\end{array}$ \\
\hline \multirow[t]{2}{*}{ NOR } & 6.3 & 8.4 & 320.1 & 276.1 & 40 & 25 \\
\hline & & & & 233.0 & 40 & 17 \\
\hline \multirow[t]{2}{*}{ CIP } & 6.1 & 8.7 & 332.1 & 314.1 & 42 & 18 \\
\hline & & & & 288.1 & 42 & 22 \\
\hline \multirow[t]{2}{*}{ PEF } & 6.3 & 7.6 & 334.1 & 316.1 & 42 & 19 \\
\hline & & & & 290.1 & 42 & 19 \\
\hline \multirow[t]{2}{*}{ LOM } & 5.8 & 9.3 & 352.1 & 308.1 & 39 & 22 \\
\hline & & & & 265.1 & 39 & 16 \\
\hline \multirow[t]{2}{*}{ ENR } & 6.3 & 8.7 & 360.2 & 316.1 & 38 & 25 \\
\hline & & & & 245.0 & 38 & 22 \\
\hline \multirow[t]{2}{*}{ OFL } & 6.1 & 8.2 & 362.1 & 318.1 & 43 & 27 \\
\hline & & & & 261.1 & 43 & 20 \\
\hline
\end{tabular}

Table S2 Matrix Effects of 6 FQs in Five kinds of Food Samples Determined by the Proposed Method $(n=6)$

\begin{tabular}{cccccccc}
\hline Samples & Concentrations & NOR & CIP & PEF & LOM & ENR & OFL \\
\hline Milk & 0.2 & $-1.9 \pm 4.6$ & $-5.3 \pm 0.4$ & $-10.7 \pm 3.6$ & $-1.8 \pm 7.7$ & $11.4 \pm 0.3$ & $-2.4 \pm 9.9$ \\
& 50 & $6.4 \pm 0.9$ & $8.2 \pm 5.3$ & $-6.6 \pm 1.8$ & $5.5 \pm 1.8$ & $2.0 \pm 6.3$ & $1.3 \pm 1.9$ \\
& 300 & $3.3 \pm 6.5$ & $2.7 \pm 5.1$ & $3.7 \pm 6.2$ & $-5.6 \pm 4.1$ & $3.5 \pm 6.4$ & $-3.3 \pm 8.2$ \\
Muscle & 0.2 & $-4.7 \pm 0.5$ & $-3.1 \pm 3.7$ & $-2.5 \pm 3.8$ & $0.4 \pm 6.1$ & $-2.2 \pm 4.2$ & $8.3 \pm 1.4$ \\
& 50 & $-9.9 \pm 2.3$ & $8.6 \pm 4.4$ & $-2.1 \pm 10.4$ & $3.3 \pm 4.2$ & $-7.8 \pm 2.2$ & $4.5 \pm 9.1$ \\
\hline
\end{tabular}




\begin{tabular}{cccccccc}
\hline \multirow{3}{*}{ Liver } & 300 & $-4.4 \pm 7.6$ & $5.4 \pm 6.2$ & $1.3 \pm 8.5$ & $5.1 \pm 6.7$ & $-4.7 \pm 8.1$ & $5.5 \pm 1.3$ \\
& 0.2 & $-10.2 \pm 2.5$ & $-4.3 \pm 5.9$ & $-3.2 \pm 6.3$ & $6.4 \pm 4.7$ & $-12.2 \pm 1.5$ & $8.1 \pm 3.7$ \\
& 50 & $-7.5 \pm 4.5$ & $9.0 \pm 4.7$ & $6.1 \pm 5.5$ & $8.8 \pm 3.2$ & $-11.2 \pm 2.1$ & $-7.9 \pm 3.7$ \\
\multirow{4}{*}{ Kidney } & 300 & $-0.3 \pm 5.0$ & $8.1 \pm 2.3$ & $-7.9 \pm 3.2$ & $6.7 \pm 2.4$ & $3.1 \pm 5.9$ & $-3.4 \pm 7.5$ \\
& 0.2 & $7.8 \pm 1.1$ & $2.1 \pm 5.8$ & $-7.1 \pm 3.2$ & $-10.1 \pm 2.2$ & $5.8 \pm 4.6$ & $-2.9 \pm 6.1$ \\
& 50 & $5.7 \pm 2.3$ & $10.3 \pm 2.2$ & $-1.2 \pm 4.3$ & $-7.9 \pm 4.8$ & $7.0 \pm 4.0$ & $-5.6 \pm 3.5$ \\
\multirow{3}{*}{ Egg } & 300 & $8.6 \pm 3.3$ & $6.7 \pm 3.4$ & $-5.3 \pm 7.1$ & $11.1 \pm 2.0$ & $2.3 \pm 3.7$ & $-4.5 \pm 7.8$ \\
& 0.2 & $-10.9 \pm 3.9$ & $4.3 \pm 1.5$ & $-5.3 \pm 5.7$ & $-5.9 \pm 4.2$ & $6.7 \pm 3.5$ & $5.4 \pm 4.0$ \\
& 50 & $-1.9 \pm 2.9$ & $5.6 \pm 8.0$ & $-8.9 \pm 1.9$ & $6.1 \pm 8.1$ & $-4.2 \pm 10.7$ & $8.0 \pm 3.4$ \\
& 300 & $2.5 \pm 3.4$ & $-6.7 \pm 2.8$ & $-7.8 \pm 1.5$ & $9.3 \pm 5.5$ & $10.0 \pm 3.5$ & $-5.2 \pm 3.0$ \\
\hline
\end{tabular}

Table S3 Intercartridge variations $(n=96)$ of the 6 FQs by the 96 -well plate $\mu$-SPE.

\begin{tabular}{|c|c|c|c|c|c|c|c|}
\hline \multirow[t]{2}{*}{ Sample } & \multirow[t]{2}{*}{ Concentration } & \multicolumn{6}{|c|}{ Analyte } \\
\hline & & NOR & CIP & PEF & LOM & ENR & OFL \\
\hline \multirow[t]{3}{*}{ Milk } & 0.2 & 7.1 & 11.9 & 12.7 & 8.3 & 10.1 & 7.7 \\
\hline & 50 & 3.4 & 7.0 & 5.8 & 9.4 & 9.6 & 5.3 \\
\hline & 300 & 5.2 & 7.6 & 8.5 & 11.4 & 7.3 & 8.7 \\
\hline \multirow[t]{2}{*}{ Meat } & 0.2 & 6.1 & 12.5 & 10.7 & 13.2 & 9.4 & 9.2 \\
\hline & 50 & 9.3 & 7.4 & 3.9 & 5.8 & 6.2 & 5.9 \\
\hline
\end{tabular}




\begin{tabular}{llllllll}
\hline \multirow{3}{*}{ Liver } & 300 & 3.6 & 6.8 & 5.9 & 6.2 & 12.1 & 8.3 \\
& 0.2 & 13.0 & 10.7 & 8.4 & 9.9 & 6.9 & 10.4 \\
& 50 & 7.9 & 8.3 & 11.2 & 9.2 & 8.6 & 9.8 \\
\multirow{4}{*}{ Kidney } & 300 & 12.9 & 7.8 & 9.5 & 12.1 & 10.5 & 9.7 \\
& 0.2 & 8.7 & 9.2 & 12.9 & 7.9 & 5.8 & 10.1 \\
& 50 & 7.5 & 8.6 & 13.3 & 9.0 & 8.5 & 9.9 \\
Egg & 300 & 10.7 & 8.1 & 11.0 & 10.7 & 12.4 & 5.9 \\
& 0.2 & 5.5 & 9.6 & 12.7 & 5.4 & 11.5 & 5.7 \\
& 50 & 9.9 & 12.6 & 7.7 & 5.7 & 7.2 & 8.1 \\
& 300 & 13.4 & 7.8 & 9.1 & 8.7 & 9.2 & 11.9 \\
\hline
\end{tabular}

Table S4 Comparison results of the proposed method with other methods.

\begin{tabular}{|c|c|c|c|c|c|c|c|c|c|}
\hline Method & Sample & $\begin{array}{l}\text { Type and volume of } \\
\text { Extraction solvent }\end{array}$ & adsorbent & $\begin{array}{l}\text { Adsorbent } \\
\text { amount }\end{array}$ & $\begin{array}{l}\text { Organic solvent } \\
\text { consumption }\end{array}$ & $\begin{array}{l}\text { Sample preparation } \\
\text { time }\end{array}$ & $\operatorname{LOD}(\mu \mathrm{g} / \mathrm{kg})$ & Recovery (\%) & Reference \\
\hline $\begin{array}{l}\text { SPME- } \\
\text { HPLC/MS-MS }\end{array}$ & Milk, honey (2 g) & Add water directly & $\begin{array}{l}\text { poly(AP-co-DB/ED) } \\
\text { monolith }\end{array}$ & - & $2.5 \mathrm{~mL}$ & $80 \mathrm{~min}$ & $\begin{array}{l}0.0010- \\
0.0037\end{array}$ & $74.5 \%-116 \%$ & {$[30]$} \\
\hline $\begin{array}{l}\text { SPE-HPLC/MS- } \\
\text { MS }\end{array}$ & Powdered milk (-) & $6.0 \mathrm{~mL} 20 \% \mathrm{TCA} / \mathrm{ACN}$ & Oasis HLB & $500 \mathrm{mg}$ & $22 \mathrm{~mL}$ & $>200$ min & $0.04-0.52$ & $84 \%$ & {$[31]$} \\
\hline $\begin{array}{l}\text { SPE-HPLC/MS- } \\
\text { MS }\end{array}$ & Milk (1 g) & $\begin{array}{l}2 \mathrm{~mL} 5 \% \text { acetic acid in } \\
\text { acetonitrile }+5 \mathrm{mLn} \text {-hexane }\end{array}$ & $\mathrm{C} 18$ & - & $10.5 \mathrm{~mL}$ & $>45 \mathrm{~min}$ & 0.2 & $85.6 \%-102.8 \%$ & [32] \\
\hline DSPE-HPLC & $\begin{array}{l}\text { Muscle, egg, milk } \\
(2 \mathrm{~g})\end{array}$ & $\begin{array}{l}10 \mathrm{~mL} \text { acetonitrile for } \\
\text { muscle } 10 \mathrm{~mL} \\
\text { trichloroacetic acid/ } \\
\text { methanol }(2: 8, \mathrm{v} / \mathrm{v}) \text { for milk } \\
\text { and egg }\end{array}$ & Magnetic graphene & $5 \mathrm{mg}$ & $12 \mathrm{~mL}$ & $130 \mathrm{~min}$ & $0.05-0.3$ & $82.4 \%-108.5 \%$ & [33] \\
\hline
\end{tabular}




\begin{tabular}{|c|c|c|c|c|c|c|c|c|c|}
\hline SBSE-HPLC & Meat (2 g) & $\begin{array}{l}10 \mathrm{~mL} \text { acetonitrile/ } \\
\text { trichloroacetic acid (7:3, } \\
\mathrm{v} / \mathrm{v})\end{array}$ & $\begin{array}{l}\text { molecularly imprinted } \\
\text { polymer }\end{array}$ & - & $15 \mathrm{~mL}$ & $105 \mathrm{~min}$ & $0.1-0.3$ & $67.4 \%-99.0 \%$ & [34] \\
\hline SBSE-HPLC & $\operatorname{Egg}(5 \mathrm{~g})$ & $\begin{array}{l}15 \mathrm{~mL} \text { of } 10(\mathrm{wt} \%) \\
\text { trichloroacetic acid }\end{array}$ & $\begin{array}{l}\text { Anionic surfactant- } \\
\mathrm{Fe}_{3} \mathrm{O}_{4} / \mathrm{SiO}_{2} \text { nanoparticles }\end{array}$ & $20 \mathrm{mg}$ & $21 \mathrm{~mL}$ & $60 \mathrm{~min}$ & 2.9 & $85 \%-94 \%$ & [35] \\
\hline SPE-UPLC & $\begin{array}{l}\text { Honey, milk ( } 5.0 \\
\text { g) }\end{array}$ & $\begin{array}{l}30 \mathrm{~mL} \text { acetonitrile }+45 \mathrm{~mL} \\
\text { n-hexane saturated with } \\
\text { acetonitrile for milk; Add } \\
\text { water directly for honey }\end{array}$ & HLB & $500 \mathrm{mg}$ & $\begin{array}{l}85 \mathrm{~mL} \text { for milk; } \\
10 \mathrm{~mL} \text { for honey }\end{array}$ & $\begin{array}{l}>230 \quad \min \text { for } \\
\text { milk; }>50 \text { min for } \\
\text { honey }\end{array}$ & 1 & $81.4 \% \sim 94.6 \%$ & {$[36]$} \\
\hline dSPE-LC & Honey $(0.5 \mathrm{~g})$ & $\begin{array}{l}4 \mathrm{~mL} \text { phosphate buffer } \\
\text { solution }\end{array}$ & $\begin{array}{l}\text { polyaniline-coated } \mathrm{SiO}_{2} \\
\text { nanofiber }\end{array}$ & $20 \mathrm{mg}$ & $1 \mathrm{~mL}$ & $12 \mathrm{~min}$ & $0.1-1.3$ & $81.4 \%-118.1 \%$ & {$[37]$} \\
\hline SPE-HPLC & Milk (2 mL) & $\begin{array}{l}2 \mathrm{~mL} 10 \mathrm{mMNH} 4 \mathrm{Ac}(\mathrm{pH} \\
5.0)\end{array}$ & $\begin{array}{l}\text { magnetic molecularly } \\
\text { imprinted polymers }\end{array}$ & $20 \mathrm{mg}$ & $3 \mathrm{~mL}$ & $>20 \mathrm{~min}$ & $1.8-3.2$ & $94.0 \%-124.4 \%$ & {$[38]$} \\
\hline $\begin{array}{l}\text { 96-well plate } \mu \text { - } \\
\text { SPE-HPLC/MS- } \\
\text { MS }\end{array}$ & $\begin{array}{l}\text { Muscle, liver, } \\
\text { kidney, milk, egg } \\
(0.5 \mathrm{~g})\end{array}$ & $\begin{array}{l}2.5 \mathrm{~mL} \text { EDTA-McIlvaine's } \\
\text { buffer }\end{array}$ & SPANI/PAN NFsM & $5 \mathrm{mg}$ & $0.7 \mathrm{~mL}$ & $<0.2 \mathrm{~min}$ & $0.012-0.06$ & $90.0 \%-110.7 \%$ & This work \\
\hline
\end{tabular}


Table S5 Detection Results of Real Samples

\begin{tabular}{|c|c|c|c|c|c|c|c|}
\hline & \multicolumn{7}{|c|}{ Concentrations $(\mu \mathrm{g} / \mathrm{L})(\%, \mathrm{RSD})$} \\
\hline & & NOR & CIP & PEF & LOM & ENR & OFL \\
\hline \multirow[t]{6}{*}{ Milk } & Brand 1 & n. d & n. $\mathrm{d}$ & n. d & $<\mathrm{LOQ}$ & n. d & n. d \\
\hline & Brand 2 & n. d & n. d & n. d & n. d & n. d & n. d \\
\hline & Brand 3 & n. d & n. d & n. d & n. $d$ & n. $d$ & n. $d$ \\
\hline & Brand 4 & $<\mathrm{LOQ}$ & n. $d$ & n. d & n. $d$ & n. $d$ & n. $d$ \\
\hline & Brand 5 & n. d & n. $d$ & n. d & n. $d$ & n. $d$ & n. $d$ \\
\hline & Brand 6 & n. d & n. d & n. $\mathrm{d}$ & n. $d$ & n. d & n. d \\
\hline \multirow[t]{6}{*}{ Muscle } & Chicken 1 & n. $\mathrm{d}$ & n. $d$ & n. d & n. $\mathrm{d}$ & $<\mathrm{LOQ}$ & n. $\mathrm{d}$ \\
\hline & Chicken 2 & n. d & n. $\mathrm{d}$ & n. d & n. d & n. d & n. $\mathrm{d}$ \\
\hline & Chicken 3 & n. d & n. d & n. $\mathrm{d}$ & n. d & n. d & n. d \\
\hline & Pork 1 & n. d & n. d & n. $\mathrm{d}$ & n. d & n. d & $<\mathrm{LOQ}$ \\
\hline & Pork 2 & n. d & n. d & n. d & n. d & n. $\mathrm{d}$ & n. d \\
\hline & Pork 3 & n. d & n. $\mathrm{d}$ & n. d & n. $\mathrm{d}$ & n. $\mathrm{d}$ & n. $\mathrm{d}$ \\
\hline \multirow[t]{3}{*}{ Liver } & Liver 1 & n. d & n. d & n. d & n. d & $3.4 \mu \mathrm{g} / \mathrm{kg}(5.6)$ & n. d \\
\hline & Liver 2 & n. $\mathrm{d}$ & n. d & n. d & n. $\mathrm{d}$ & n. d & n. d \\
\hline & Liver 3 & n. d & $<\mathrm{LOQ}$ & n. $\mathrm{d}$ & n. d & n. $d$ & n. $\mathrm{d}$ \\
\hline \multirow[t]{3}{*}{ Kidney } & Kidney 1 & n. d & n. d & n. d & n. d & n. d & n. d \\
\hline & Kidney 2 & n. $d$ & n. $\mathrm{d}$ & n. $\mathrm{d}$ & n. d & n. d & n. $\mathrm{d}$ \\
\hline & Kidney 3 & n. d & $1.2 \mu \mathrm{g} / \mathrm{kg}(3.4)$ & n. d & n. $\mathrm{d}$ & n. $\mathrm{d}$ & n. $\mathrm{d}$ \\
\hline \multirow[t]{5}{*}{ Egg } & Native egg 1 & n. d & n. d & n. d & n. d & n. $\mathrm{d}$ & n. d \\
\hline & Native egg 2 & n. d & n. d & n. d & n. d & n. $\mathrm{d}$ & n. d \\
\hline & Native egg 3 & n. $d$ & n. d & n. $\mathrm{d}$ & n. d & n. d & n. d \\
\hline & Ordinary egg 1 & n. d & n. $\mathrm{d}$ & n. $\mathrm{d}$ & n. d & $0.49 \mu \mathrm{g} / \mathrm{kg}(2.5)$ & n. d \\
\hline & Ordinary egg 2 & n. d & $<\mathrm{LOQ}$ & n. d & n. d & n. d & n. d \\
\hline
\end{tabular}


